\title{
Presenze del potere centrale nelle città della Gallia Cisalpina: i documenti epigrafici
}

Gino Bandelli

\section{Trieste}

bandelgi@units.it

\section{Fecha recepción 22.02.2016 / Fecha aceptación 22.06.2016}

\section{Riassunto}

Dopo un' introduzione di carattere generale sull' assetto amministrativo della Gallia Cisalpina durante il periodo repubblicano, il contributo elenca i magistrati romani documentati epigraficamente in vari centri coloniari della provincia: Manio Acilio Glabrione, cos. 191, a Luna (due volte); Lucio Mummio, cos. 146, a Parma; Marco Claudio Marcello, cos. II 155, a Luna; Gaio Sempronio Tudita-

\begin{abstract}
After a short introduction about the administrative organization of Gallia Cisalpina during the Republican period, this paper gives a list of the epigraphically documented Roman magistrates in various colonial centres of the province: Manius Acilius Glabrio, cos. 191, in Luna (twice); Lucius Mummius, cos. 146, in Parma; Marcus Claudius Marcellus, cos. II 155, in Luna; Gaius Sempronius Tuditanus, cos. 129,
\end{abstract}


no, cos. 129, ad Aquileia (due volte); Lucio Manlio Acidino, triu(m)vir Aquileiae coloniae deducundae 181, ad Aquileia; Tito Annio (Losco), tri(um)vir 169, ad Aquileia; Gaio Aurelio Cotta, pro cos. 74, ad Aquileia; Gaio Appulleio Tappone, pr. ?, ad Aquileia. La presenza in Ariminum di un' iscrizione celebrativa di Gaio Mario è considerata dubbia. Nessuna attestazione epigrafica di magistrati della Repubblica romana proviene da centri indigeni.

\section{Parole chiave}

Cisalpina repubblicana, Magistrati romani, Prosopografia epigrafica. in Aquileia (twice); Lucius Manlius Acidinus, triu $(m)$ vir Aquileiae coloniae deducundae 181, in Aquileia; Titus Annius (Luscus), tri(um)vir 169, in Aquileia; Gaius Aurelius Cotta, pro cos. 74, in Aquileia; Gaius Appulleius Tappo, pr. ?, in Aquileia. The existence in Ariminum of an inscription celebrating Gaius Marius is considered doubtful. There is also no epigraphic evidence of magistrates of the Roman Republic deriving from indigenous centres.

\section{Key words}

Republican Cisalpina, Roman magistrates, Epigraphic prosopography. 
Nella storia della Gallia Cisalpina possiamo distinguere due periodi: I) quello in cui, nella sua totalità (sotto le denominazioni di Gallia o Italia) ovvero per territori specifici (dei Galli, dei Ligures, degli Histri), essa fu provincia nel senso originario della parola, cioè quale àmbito di competenza di un magistrato con imperium ${ }^{1}$; II) quello successivo alla sua costituzione come provincia ordinaria, delimitata rispetto all' Italia dall' Arno sul versante tirrenico e dal Rubicone sul versante adriatico, rispetto alla Gallia Narbonense dal Varo, rispetto all' Illirico dal Timavo ${ }^{2}$.

Sulla cronologia della redactio in formam provinciae le opinioni sono molte e discordi: l'alternativa più probabile mi sembra quella tra la proposta, venuta da Filippo Càssola, di un anno imprecisabile dal $143 \mathrm{al} 95$ a. C. ${ }^{3}$ e la proposta, già fatta da Theodor Mommsen e ripresa infine da Giorgio Luraschi e da altri, dell'epoca della dittatura sillana o poco dopo ${ }^{4}$. Quanto al periodo conclusivo di tale ordinamento, le date fondamentali sono il 49 a. C., in cui la cittadinanza romana fu estesa a tutti gli abitanti del suo territorio ${ }^{5}$, e il $42-41$ a. C., in cui essa fu inglobata nell' Italia ${ }^{6}$.

Nella prospettiva di questa ricerca tralascerò di analizzare il ruolo avuto dai centri più importanti della Gallia Cisalpina, tanto indigeni quanto coloniari, nelle varie fasi della conquista

1. P. A. Brunt, Italian Manpower, 225 B. C. - 14 A. D., Oxford 1971, 567-569 (567: «...'Italy’ or Cisalpina including Histria or Liguria; 'Italy' in fact meant the north»); F. Càssola, "La colonizzazione romana della Transpadana”, in W. Eck e H. Galsterer (Edd.), Die Stadt in Oberitalien und in den nordwestlichen Provinzen des Römischen Reiches, Deutsch-Italienisches Kolloquium im italienischen Kulturinstitut Köln (1989), Mainz am Rhein 1991, 17-44, in part. 41-43.

2. U. Laffi, "La provincia della Gallia Cisalpina", in Athenaeum, 80, 1992, 5-23, in part. 6-7 = U. Laffi, Studi di storia romana e di diritto, Roma 2001, 211-212.

3. Càssola, loc. cit., 1991, 30-40 e 43-44.

4. G. Luraschi, Foedus Ius Latii Civitas. Aspetti costituzionali della romanizzazione in Transpadana, Padova 1979, 179-189.

5. Dio 41.36.3; Laffi, loc. cit., 1992, 11, nota 25 = Laffi, loc. cit., 2001, 217, nota 25.

6. Fonti e bibliografia in Laffi, loc. cit., 1992, 11, nota 26 = Laffi, loc. cit., 2001, 218, nota 26. 
romana. Rilevo soltanto che uno di questi, Ariminum, fu considerato a lungo sinonimo della provincia $;$; e, quanto al resto, rimando alla trattazione sistematica di Raymond Chevallier ${ }^{8}$.

Non prenderò in esame nemmeno lo sviluppo di fenomeni come le deduzioni di coloniae Latinae e di coloniae civium Romanorum e le assegnazioni viritane ed i relativi conciliabula, sui quali esiste una letteratura vastissima9 ${ }^{9}$, e come le costituzioni di Fora, per cui possiamo avvalerci tuttora della monografia di Eeva Ruoff Väänänen ${ }^{10}$.

Resta, comunque, un dato di fatto paradossale, se teniamo presente l' importanza della Gallia Cisalpina: cioè che della struttura del suo governo, anche dopo la costituzione della provincia ordinaria, non sappiamo quasi nulla. Che la sua capitale fosse Cremona, secondo quanto afferma Raymond Chevallier ${ }^{11}$, non risulta da nessuna fonte. Delle stationes portorii la sola documentata, non prima dell' età sillana, è quella di Aquileia ${ }^{12}$. Quanto alla distribuzione dei conventus iuridici, attestati dall' epoca di Cesare ${ }^{13}$, l' unico loro centro individuabile con certezza è, di nuovo, Aquileia, dove, come risulta da un' epigrafe di Salona, il 3 marzo del 56 a. C. il proconsole compose una vertenza che, scoppiata comunque in territorio illirico, aveva interessato in qualche modo le città greche di Issa e di Tragurion e la componente italica (di Salona?) e quella indigena (di Iader?) ${ }^{14}$.

7. Liv. 24.44.2: ... provincia Ariminum... (213 a. C.); 28.38.13: ... Ariminum - ita Galliam appellabant -... (205 a. C.); 30.1.9: ... Ariminum cum duabus legionibus... (203 a. C.); 32.1.2: ... Ariminum... (199 a. C.). Cfr., per le guerre contro i Ligures, il riferimento a Pisae: Liv. 38.35 .8 (188 a. C.); 41.5.6; 12.1 (178 a. C.; 177 a. C.); 45.16.3; 17.6 (167 a. C.).

8. R. Chevallier, La romanisation de la Celtique du Pô (essai d' histoire provinciale), vol. III, Histoire et administration, Tours 1979.

9. Fonti e bibliografia, da ultimo, in G. Bandelli, "Parma durante la Repubblica. Dalla fondazione della colonia a Cesare”, in D. Vera (Ed.), Storia di Parma, vol. II, Parma romana, Parma 2009, 180-217, passim e in G. Bandelli, "Roma e la Gallia Cisalpina dal "dopoguerra annibalico" alla guerra sociale (201 a. C. - 89 a. C.)", in P. Piana Agostinetti (Ed.), Celti d' Italia, Atti del Convegno internazionale (Roma 2010), in corso di stampa, passim.

10. E. Ruoff Väänänen, Studies on the Italian Fora, Historia - Einzelschriften, 32, Wiesbaden 1978. Nei limiti di questo contributo non posso elencare gli studi relativi ai singoli centri.

11. Chevallier, op. cit., 1979, 96.

12. Fonti e bibliografia, da ultimo, in G. Bandelli, "Stranieri ad Aquileia in età repubblicana", in J. M. Iglesias Gil e A. Ruiz Gutiérrez (Edd.), Viajes y cambios de residencia en el mundo romano, [Atti del Convegno internazionale (Santander 2011)], Santander 2011, 23-45, in part. 27, note 35-36 e 31-32, note 67-74.

13. Caes., B. G. 1.54.3: ... ipse in citeriorem Galliam ad conventus agendos profectus est; 5.1.5: Ipse conventibus Galliae citerioris peractis in Illyricum proficiscitur...; 6.44.3: ... in Italiam ad conventus agendos profectus est; 7. 1. 1: ... in Italiam ad conventus agendos proficiscitur; Hirt., B. G. 8.23.3: ... Caesare in Gallia citeriore ius dicente...; Suet., Div. Iul. 30.1: ... conventibusque peractis Ravennae substitit...; 56.6: ... cum ex citeriore Gallia conventibus peractis ad exercitum rediret....

14. R. K. Sherk, Roman documents from the Greek East. Senatus consulta and epistulae to the age of Augustus, Baltimore, Maryland 1969, 139-142, nr. 24; R. K. Sherk, Rome and the Greek East to the death of Augustus, Cambridge 1984, 96, nr. 77; Ph. Culham, "Romans, Greeks, and Delmatae: Reconstructing the Context of RDGE 24”, in Classical Antiquity, 12, nr. 1, 1993, 51-64. Da ultimo: M. Šašel Kos, Appian and Illyricum, Ljubljana 2005, 342-343; D. Dzino, Illyricum in Roman politics, 229 BC - AD 68, New York 2010, 87-89; S. Sisani, “Tergeste e le "colonie" cesariane della Gallia Togata (in margine a B. G. 8.24.3)", in 


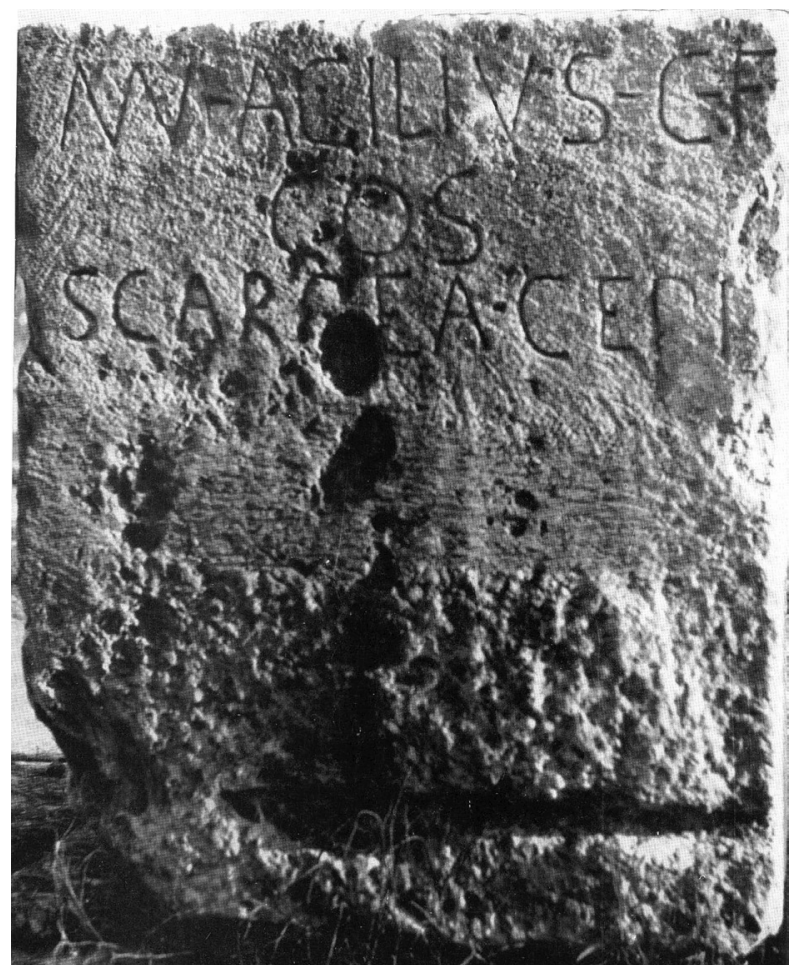

Fig. 1. Da Imagines 140.

In tale situazione limiterò l' indagine a una serie di tituli che documentano la presenza in città diverse della provincia di esponenti del potere centrale.

Una buona parte di questi è compresa nel II secolo a. C.

I due più antichi provengono da Luna (Figg. 1,2). Si tratta di basi che recano le seguenti epigrafi:
M(anius) Acilius $C($ ai) f(ilius)
$M($ anius) Acilius $C($ ai) f(ilius)
$\operatorname{co}(n) s(u l)$
$\operatorname{co}(n) s(u l)$
Scarpea cepi $(t)^{15}$.
Heracelea cepi $(t)^{16}$.

A. Giovannini (Ed.), Trieste e l' Istria. Incontri a tema per la diffusione della storia e del patrimonio culturale, in corso di stampa, note 159-172.

15. ILLRP 321 a; Imagines 140; CIL I², 2926, F. Frasson, Le epigrafi di Luni romana, I, Alessandria 2013, 429-434.

16. M. G. Angeli Bertinelli, "Un titulus inedito di M'. Acilio Glabrione, da Luni", in Mélanges de l'École Française de Rome. Antiquité, 105, nr. 1, 1993, 7-31 = M. G. Angeli Bertinelli, Lunensia antiqua, Roma 2011, 253-275, figg. 52, 102, 103, 104; AÉ 1993, 643. 


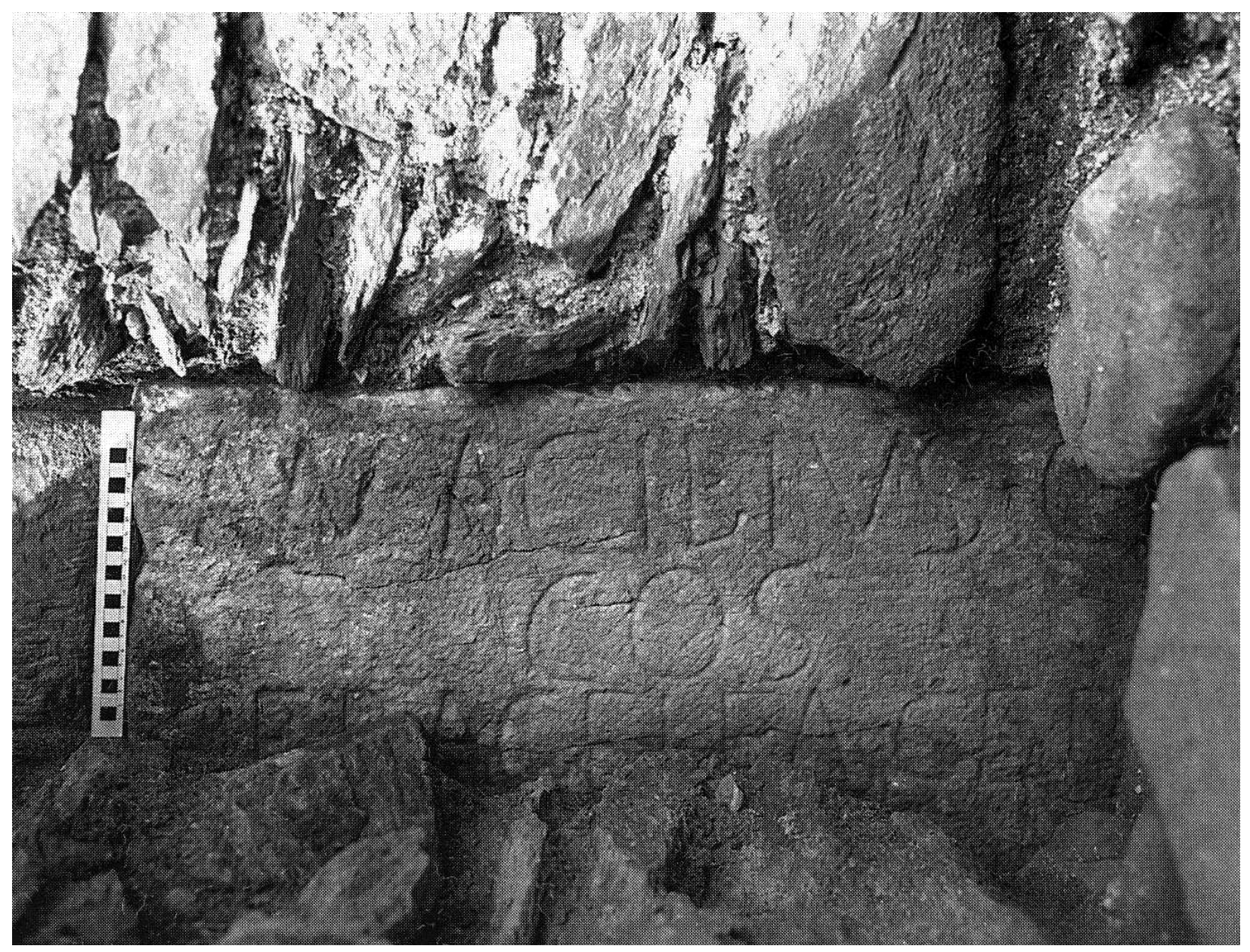

Fig. 2. Da Angeli Bertinelli 1993 = Angeli Bertinelli 2011.

Dai testi emerge che Manio Acilio Glabrione, console del 191 a. C., fece dono alla popolazione locale di due statue non meglio identificabili acquisite, durante la fase europea della guerra contro Antioco III, nel saccheggio delle città di Scarfea (Locride Opunzia o Epicnemidia) e di Eraclea Trachinia (Malide).

In rapporto ai dati finora disponibili tale iniziativa risulta avere dei precedenti a Roma (dedica di Marco Claudio Marcello con formula Hinnad cepit: 211 a. C.?) ${ }^{17}$ ed a Praeneste

17. CIL I², 608 (e p. 918); ILLRP 295. 
(dedica di Lucio Quinzio Flaminino con formula [Le]ucado cepit: 192 a. C.) ${ }^{18}$, ma non in ambiente provinciale. Il fatto che gl' interventi di Glabrione avessero luogo prima della fondazione della colonia civium Romanorum, dedotta nel 177 a. C., dà la misura della rilevanza del portus Lunae, da cui nel 195 a. C. era partito verso la penisola iberica il console Marco Porcio Catone ${ }^{19}$; e offre probabilmente una chiave di lettura di segno anticatoniano per l' iniziativa del console del 191 a. C., seguace di Publio Cornelio Scipione e avversario, dunque, del futuro Censore (che nel 190 a. C., in vista delle elezioni per tale carica, lo avrebbe accusato di appropriazione indebita di una parte della preda di guerra $)^{20}$.

Nei decenni successivi operazioni del genere si moltiplicano, riscontrandosi, oltre che in Roma, in Italia e in Grecia ${ }^{21}$, pure in province ordinarie, come la Sicilia e la Spagna Ulteriore $^{22}$. Il ripetersi del fenomeno rende perciò meno significativa la presenza, fra i comunque numerosi tituli Mummiani collegati alla fine della guerra acaica, cioè al 146 a. C. ${ }^{23}$, di uno, restitutus, pertinente a una comunità della Cisalpina, cioè alla colonia civium Romanorum di Parma (Fig. 3):

18. CIL I², 613 (e p. 918); ILLRP 321; Imagines 139. Sul fenomeno in generale: Angeli Bertinelli, loc. cit., 1993, 13-15 = Angeli Bertinelli, loc. cit., 2011, 257-259.

19. Liv. 34.8.4-5.

20. Liv. 37.57.13-14.

21. CIL I², 615 (e pp. 833 e 919); ILLRP 124 (Marco Fulvio Nobiliore, con formula Ambracia cepit: 189 a. C., da Roma). CIL I², 616 (e p. 919); ILLRP 322; Imagines 141 (Marco Fulvio Nobiliore, con formula Aetolia cepit: 189 a. C., da Tusculum). CIL I², 622 (e pp. 725 e 739); ILLRP 323; Imagines 142 (Lucio Emilio Paolo, con formula de rege Perse Macedonibusque cepet: 167 a. C., da Delfi). CIL I², 625 (e p. 920); ILLRP 326 (Publio Cornelio Scipione Emiliano, con formula Carthagine capta: 146 a. C., titulus restitutus da Marruvium Marsorum).

22. Sicilia, Himera, due tituli di Publio Cornelio Scipione Emiliano: dati e bibliografia in J.-L. Ferrary, Philhellénisme et impérialisme. Aspects idéologiques de la conquête romaine du monde hellénistique, de la seconde guerre de Macédoine à la guerre contre Mithridate, Rome 1988, 578-580. Spagna Ulteriore, Italica, un titulus restitutus mutilo attribuito originariamente a Lucio Mummio, cos. 146 (CIL II, 1119; CIL I², 630; ILLRP 331), successivamente a Lucio Emilio Paolo, cos. 168: A. M. Canto, "Un nuevo documento de Paulo Emilio en la Hispania Ulterior: CIL, I, 546 = CIL, II, 1119”, in Epigraphica, 47, 1985, 9-19; HEp 1, 1989, 545; CILA II, 2, 377, fig. 207; HEp, 4, 1994, 698; HEp 16, 2010, 534. A favore dell' identificazione primitiva: Ferrary, op. cit., 1988, 580-581, nota 29; J. Beltrán Fortes, "Luxuria helenística en la Hispania tardorrepublicana”,

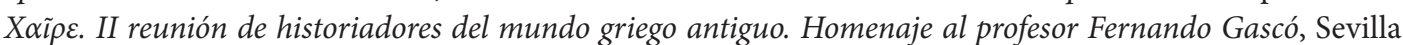
1997, 311-327, in part. 317-322; A. U. Stylow, "Fuentes epigráficas para la historia de la Hispania Ulterior en época republicana”, in E. Melchor Gil, J. Mellado Rodríguez, J. F. Rodríguez Neila (Edd.), Julio César y Corduba: tiempo y espacio en la campaña de Munda (49-45 a. C.), Actas del Simposio (Córdoba 2003), Córdoba 2005, 247-262, in part. 249, nota 7. Sono grato ad Antonio Caballos per le indicazioni che mi ha dato sul problema.

23. CIL I², 627 (e p. 921); ILLRP 327; Imagines 145 a, b. CIL I², 628 (e p. 921); ILLRP 329. CIL I², 629 (e p. 921); ILLRP 330; cfr. nota 24. CIL I², 630 (e p. 921); ILLRP 331; cfr. nota 22. CIL I², 631 (e p. 921); ILLRP 328. 


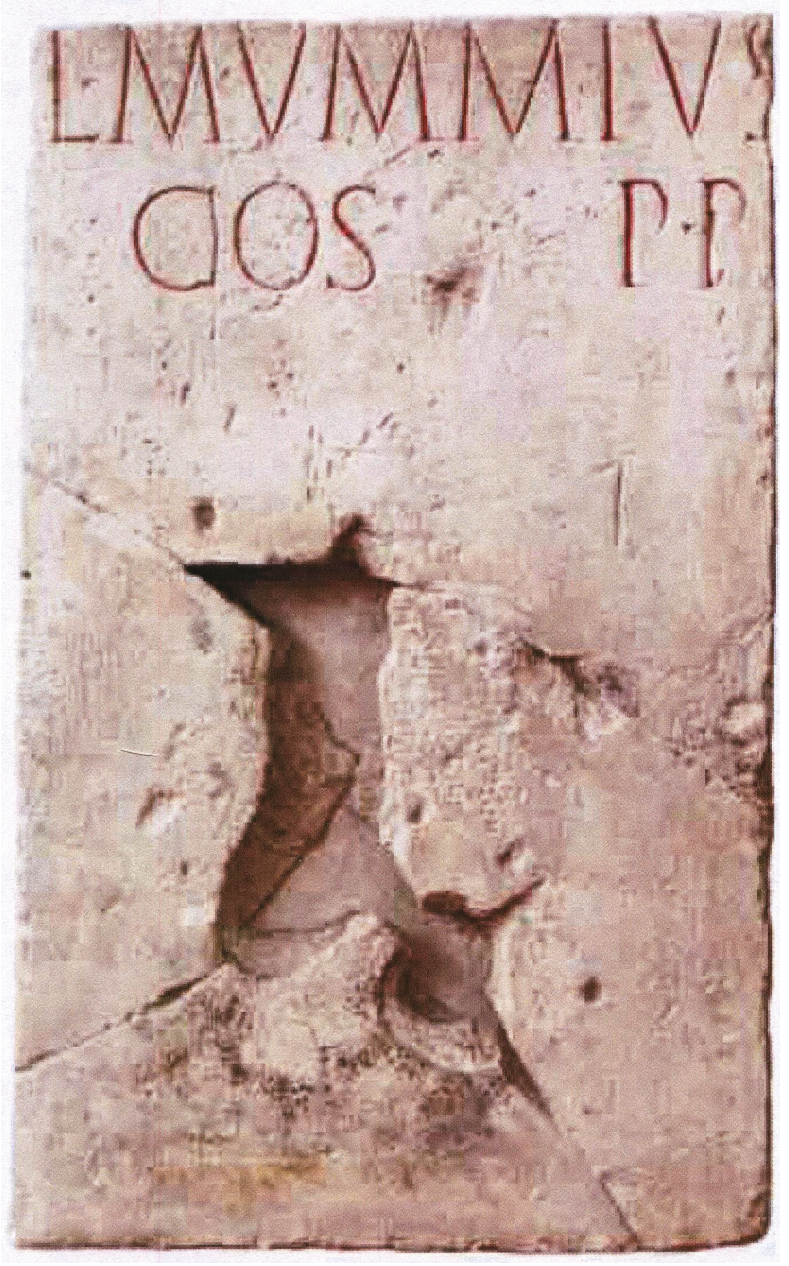

Fig. 3. Da Bandelli 2009.

\section{$L$ (ucius) Mummius \\ co(n)sul p(opulo) $P(\text { armensi })^{24}$.}

Ad un genere diverso di epigrafi riporta invece quella incisa nell'ábaco di una colonna marmorea dal Foro della colonia civium Romanorum di Luna (Fig. 4), menzionante Marco Claudio Marcello, consol iterum nel 155 a. C., e collegabile al trionfo [de - - ] us et Apua[neis] celebrato in quell' anno dal suo destinatario ${ }^{25}$. È possibile che si tratti di un gesto di autoesaltazione del magistrato; ma non pare del tutto da escludere, in alternativa, che il monumento derivi da una delibera della cittadinanza. Eccone il testo:

\section{$M$ (arcus) Claudius $M($ arci) f(ilius) Marcelus consol iterum ${ }^{26}$.}

Dall' iniziativa del rappresentante del governo centrale dipende, nell' un caso con certezza, nell' altro con probabilità, una coppia di tituli, rispettivamente da Aquileia o dintorni e dal confine orientale del suo territorio, il cui soggetto è Gaio Sempronio Tuditano, console del 129 a. C., che guidò, in prima persona o tramite dei legati, una serie di operazioni militari contro vari popoli delle regioni contigue - Carni (?), Taurisci, Istri, Giapidi e Liburni ${ }^{27}$ - e ottenne un trionfo de Iapudibus ${ }^{28}$.

Il frammento sinistro del primo documento, probabilmente un' ara, fu trovato, in situ o in reimpiego, nella frazione extra-urbana di Monastero, quello destro, in reimpiego, nel vecchio ponte sul

24. CIL I², 629 (e p. 921); ILLRP 330. Si tratterebbe di una copia del II secolo d. C. (Nel commento del CIL e delle ILLRP non viene peraltro esclusa una lettura diversa del testo originario). Da ultimo: Bandelli, $l o c$. cit., 2009, 205-206, fig. 99.

25. Fasti triumphales: InIt XIII, 1, pp. 82-83 e 557.

26. CIL I², 623 (e p. 920); ILLRP 325; Imagines 144 ; Frasson, op. cit., 2013, 67-70.

27. Fonti letterarie: Liv., Per. 59.20 (Giapidi); Plin., N. H. 3.23.129 (Istri); App., Illyr. 10.30 (Giapidi). Il coinvolgimento certo dei Taurisci e verosimile dei Carni risulta dalla parte centrale (Tauriscos C[arnosque?]) del primo verso pervenutoci del titulus metrico: nota 30.

28. Fasti triumphales: InIt XIII, 1, pp. 82-83 e 559. 


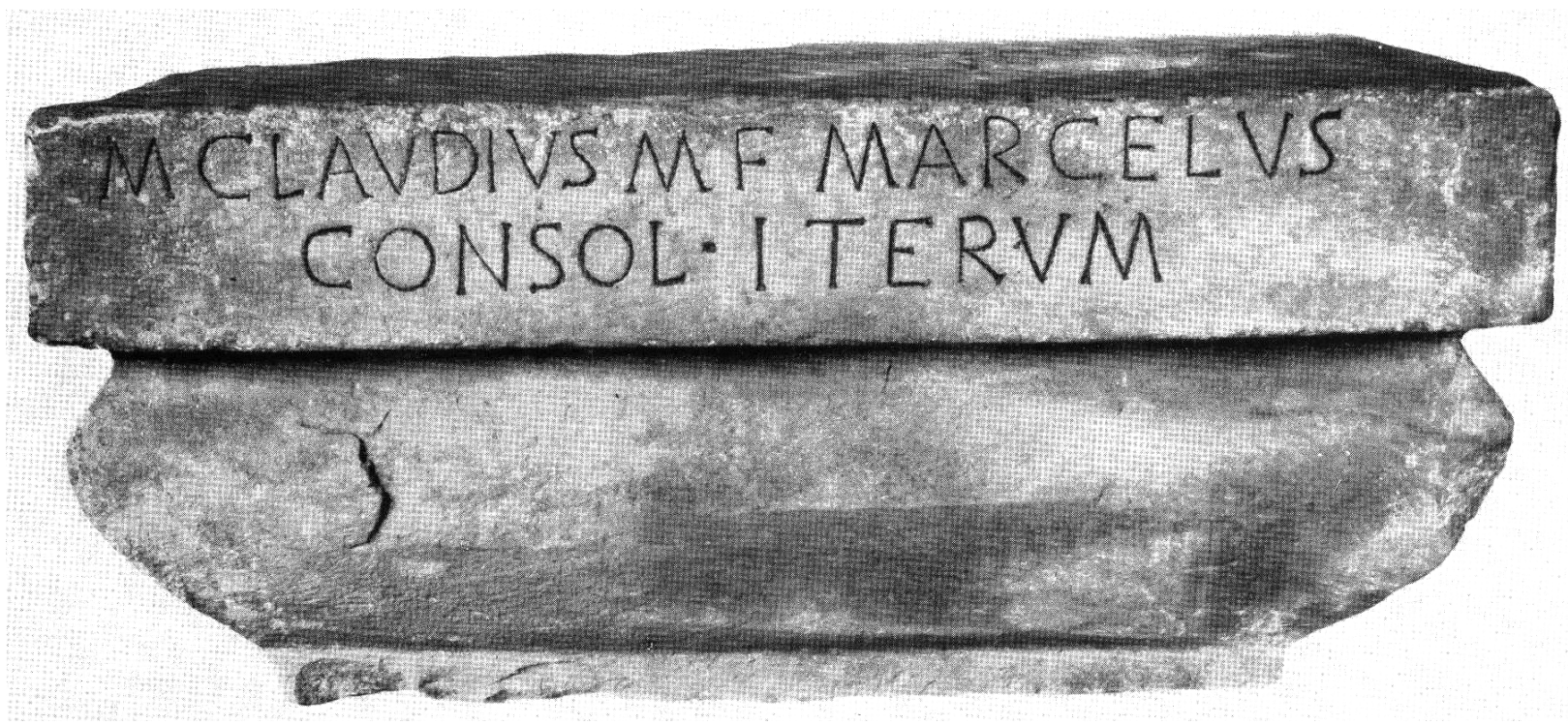

Fig. 4. Da Imagines 144.

fiume Aussa della più distante cittadina di Cervignano (Fig. 5 ${ }^{29}$. Dell' iscrizione manca la parte iniziale e rimangono sei versi in metro saturnio, che riassumono le gesta del magistrato, ma sono alquanto mutili (donde le più diverse proposte $\mathrm{d}$ ' integrazione degli editori):

$$
\begin{aligned}
& \text { [- - - - ]re et Tauriscos C[- - - - ] }
\end{aligned}
$$

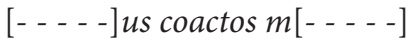

$$
\begin{aligned}
& \text { [- - - - ]r quineis qua[- - - ] ]avit } \\
& \text { [- - - - ] signeis consi[- - - - os Tuditanus } \\
& \text { [- - - - ] e egit triumpu[- . - - ] dedit Timavo } \\
& \text { [- - - - ] riaei restitu[- - - - ] reis tradit }{ }^{30} \text {. }
\end{aligned}
$$

29. Per le informazioni al riguardo cfr. i commenti di Theodor Mommsen (CIL V) e di Giovanni Brusin (InAq).

30. CIL V, 1, 39*; 2, 8270; CIL I², 652 (e pp. 725, 834, 926); CLE 1859; InIt XIII, 3, 90; InIt X, 4, 317 b, c; ILLRP 335; Imagines 147; InAq 28; G. Lettich, Itinerari epigrafici aquileiesi. Guida alle iscrizioni esposte del Museo Archeologico Nazionale di Aquileia, Trieste 2003, 40, nr. 32; cfr. G. Bandelli, Ricerche sulla colonizzazione romana della Gallia Cisalpina. Le fasi iniziali e il caso aquileiese, Roma 1988, 97, nr. 2 e G. Bandelli, "Contributo all' interpretazione del cosiddetto elogium di C. Sempronio Tuditano", in [M. Mirabella Roberti (Ed.)], Aquileia repubblicana e augustea, Atti della XIX Settimana di Studi Aquileiesi (1988), Udine 1989, 111-131 (dove alcune ipotesi ulteriori d' integrazione testuale). Una rassegna della bibliografia più recente in G. Bandelli, “Trieste e l' Istria dall' avvio della conquista romana (221-220 a. C.) allo spostamento del confine italico sul fiume 


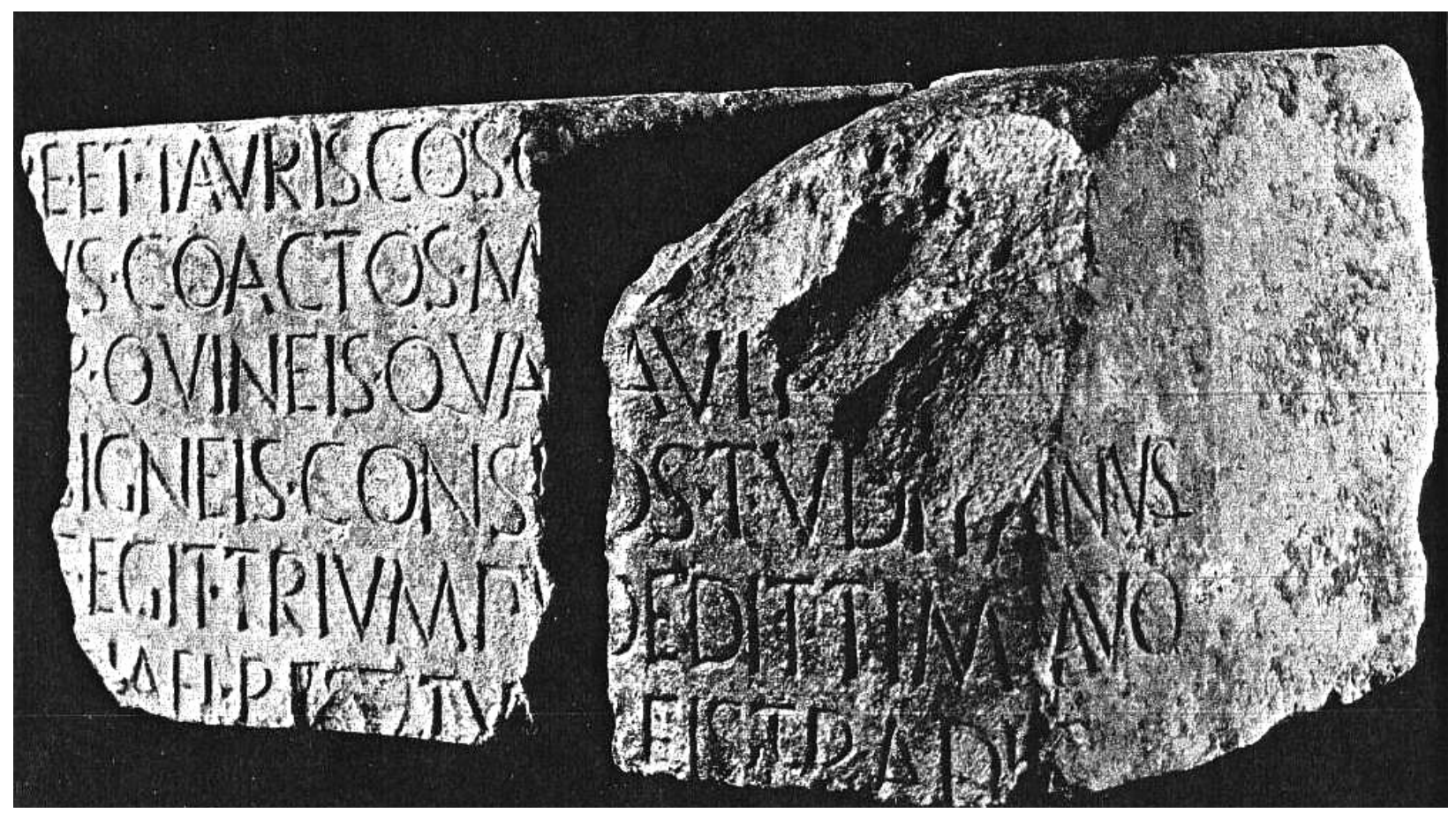

Fig. 5. Da Imagines 147.

Circa la provenienza originaria del titulus (in cui si menziona, fra l' altro, la celebrazione del trionfo e una dedica al Timavo, deità fluviale indigena), la teoria più ragionevole sembra che questo fosse compreso nell' àmbito di un preesistente santuario edificato nella suddetta località di Monastero, che Federica Fontana data fra gli Anni 170 e 160 a. C. e collega al trionfo di Gaio Claudio Pulcro, cos. 177 a. C., nella seconda (o terza) guerra istrica ${ }^{31}$. Nessun

Arsia (18/12 o 6/4 a. C.). Aspetti politici, militari, amministrativi, economici, sociali", in Giovannini (Ed.), op. cit., in corso di stampa. Adde: M. Tarpin, "Inscriptions républicaines et triomphe: rituel et obligations sociales", in C. Deroux (Ed.), Corolla Epigraphica. Hommages au professeur Yves Burnand, vol. 2, Bruxelles 2011, 683-699, in part. 688-689.

31. F. Fontana, "Rappresentazione di una vittoria nel frontone fittile di Monastero", in Cicli figurativi di terracotta in età repubblicana, Atti del Convegno internazionale (Chianciano 1992), Parte seconda, in Ostraka, vol. III, nr. 1, 1994, 175-188; F. Fontana, "Due casi di committenza sacra ad Aquileia", in M. Cébeillac-Gervasoni (Ed.), Les élites municipales de l' Italie péninsulaire des Gracques à Néron, Actes de la table ronde internationale de Clermont-Ferrand (1991), Naples - Rome 1996, 227-246, in part. 228-240; F. Fontana, I culti di Aquileia repubblicana. Aspetti della politica religiosa in Gallia Cisalpina tra il III e il II sec. a. C., Roma 1997, 27-51 e 203-208, figg. 32-39; M. Verzár-Bass, "Osservazioni sui luoghi e monumenti di vittoria militare nell' Adriatico nord-orientale", in Atti e Memorie della Società Istriana di Archeologia e Storia Patria, 102, 2002, 49-74, in part. 54-56; F. Fontana, "Topografia del sacro ad Aquileia: alcuni spunti”, 
indizio, se non quello genericissimo che materiali del Foro di Aquileia vennero trasferiti a Monastero già in epoca tardo-antica ${ }^{32}$, riconduce alla piazza principale della colonia latina. Un contesto templare suggerisce comunque la natura stessa del documento, che non è un elogium, come talvolta si ripete ${ }^{33}$, ma qualcosa di assimilabile ad una tabula triumphalis, come fu visto già da Franz Bücheler e Hermann Dessau e ribadito poi da Maria José Strazzulla Rusconi e da me: la collocazione consueta di tal genere di epigrafi era, appunto, in edifici sacri ${ }^{34}$. Dal nostro punto di vista risulta dunque di particolare interesse il fatto che, a differenza delle attestazioni precedenti di tabulae triumphales, tutte da Roma, quello di Aquileia è il primo, e finora unico, esempio della categoria posto in territorio provinciale.

Quanto al secondo monumento del Tuditano, attualmente irreperibile, si tratta probabilmente di una base, anch'essa frammentaria. La sua provenienza non da Aquileia, ma dal santuario del Timavo localizzato in vicinanza delle risorgive carsiche del fiume, site una ventina di chilometri a oriente della colonia latina, è ovvia: lo si trovò reimpiegato in un bastione del castello di Duino, che sorge meno di due chilometri a sud-est delle risorgive medesime ${ }^{35}$. Il testo è il seguente:

$$
\begin{aligned}
& {[C(\text { aius })] \text { Sempronius } C(\text { ai }) f(\text { ilius })} \\
& {[T] \text { uditanus co }(n) s(u l)} \\
& -\ldots--^{36} \text {. }
\end{aligned}
$$

in G. Cuscito e M. Verzár-Bass (Edd.), Aquileia dalle origini alla costituzione del Ducato longobardo. La cultura artistica in età romana. Topografia - Urbanistica - Edilizia pubblica, Atti della XXXIV Settimana di Studi Aquileiesi (2003), Trieste 2004, 401-424, in part. 403-404 e 411-413; F. Fontana, "Testimonianze di culti in area nord-adriatica: il caso di Apollo e Diana", in F. Lenzi (Ed.), Rimini e l'Adriatico nell' età delle guerre puniche, Atti del Convegno Internazionale di Studi (Rimini 2004), Bologna 2006, 313-331, in part. 321; F. Fontana, "I culti della romanizzazione", in G. Cuscito (Ed.), Aspetti e problemi della romanizzazione. Venetia, Histria e arco alpino orientale, Atti della XXXIX Settimana di Studi Aquileiesi (2008), Trieste 2009, 297-308, in part. 304. Secondo M. Verzár-Bass, "Riflessioni sui santuari extraurbani della colonia latina di Aquileia", in $\Delta \dot{v} v \alpha \sigma \theta \alpha \iota \delta ı \delta \alpha ́ \sigma \kappa \varepsilon v v$. Studi in onore di Filippo Càssola, Trieste 2006, 423-438, in part. 425: «il monumento... poteva trovarsi sia a Monastero, sia nella zona dell'Aussa o in un altro posto ancora». Da ultimo: E. Murgia, Culti e romanizzazione. Resistenze, continuità, trasformazioni, Trieste 2013, 267-272.

32. Bandelli, "Trieste e l' Istria", loc. cit., in corso di stampa, nota 13.

33. Per la bibliografia sul problema cfr. Bandelli, loc. cit., 1989, 113-127 e Bandelli, “Trieste e l' Istria”, loc. cit., in corso di stampa, note 27-30. Da ultimo: Tarpin, loc. cit., 2011, 688-689.

34. M. J. Strazzulla Rusconi, "L'edilizia templare ed i programmi decorativi in età repubblicana", in La città nell' Italia settentrionale in età romana. Morfologie, strutture e funzionamento dei centri urbani delle Regiones X e XI, Atti del Convegno (Trieste 1987), Trieste - Roma 1990, 279-304, in part. 296-297 e Bandelli, loc. cit., 1989, 114-116.

35. A proposito del santuario in generale cfr., ora, Murgia, op. cit., 2013, 293-296. Una completa ricostruzione delle disavventure occorse all'epigrafe è presentata da M. Chiabà, "Lo strano caso dell' iscrizione frammentaria di Gaio Sempronio Tuditano, cos. 129 a. C., da Duino (agro di Aquileia)", in Epigraphica, 75, 2013, 107-125.

36. CIL I I 2503 (e pp. 926-927); InIt X, 4, 317 (dove un indebito collegamento con l' iscrizione trionfale); ILLRP 334; Imagines 148; G. Alföldy, Römische Statuen in Venetia et Histria. Epigraphische Quellen, 


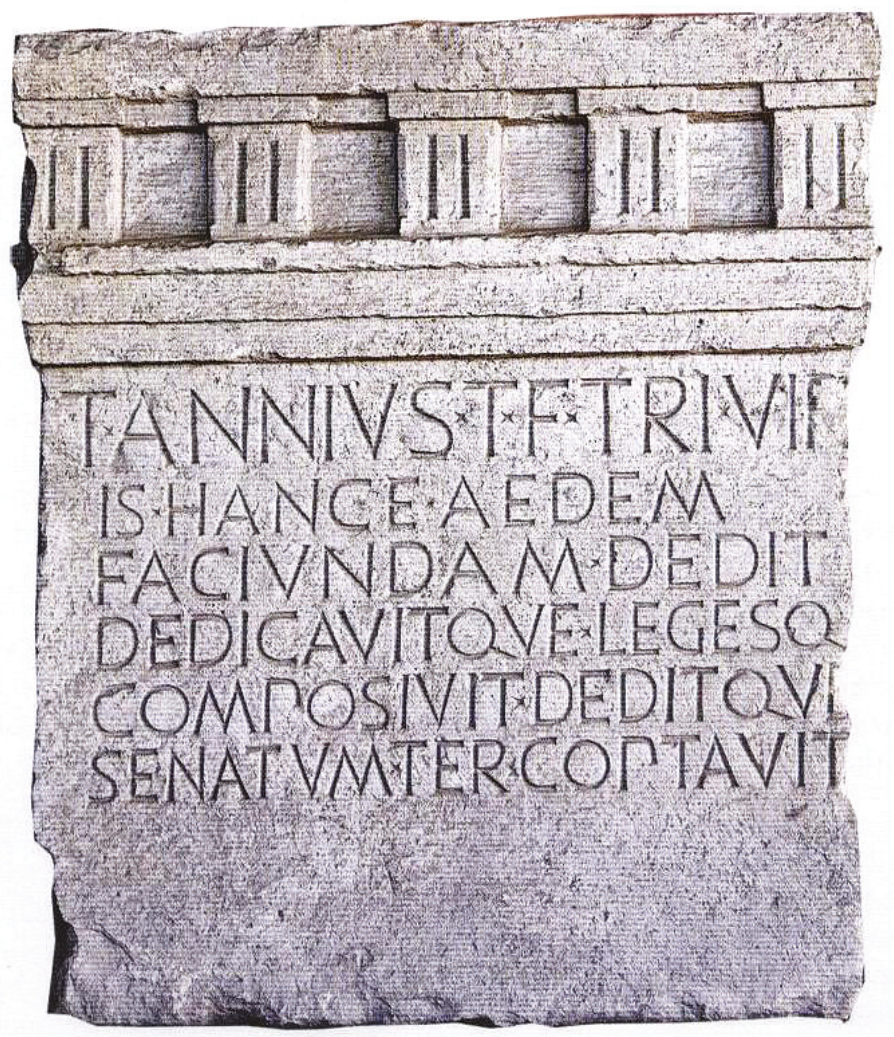

Fig. 6. Da Chiabà 2009.

Che il titulus coincida con quello posto dal console nel territorio di uno dei popoli attaccati ${ }^{37}$, di cui Plinio il Vecchio riporta una parte dell'epigrafe (Tuditanus, qui domuit Histros, in statua sua ibi inscripsit: AB AQVILEIA AD TITIVM FLVMEN STADIA $M<M>)^{38}$, è proponibile, ma non certo: considerato che l' ibi pare alludere a una località già compresa nella penisola istriana, il testo citato nella Naturalis historia potrebbe riferirsi ad un terzo monumento.

Anche due altre testimonianze del II secolo a. C., databili con minore precisione, provengono da Aquileia.

Heidelberg 1984, 87, nr. 43 (dove un altro collegamento con l' iscrizione trionfale); Bandelli, loc. cit., 1989, 97, nr. 3 (dove ulteriore bibliografia).

37. Sulla complessità delle azioni militari del 129 a. C. cfr. supra, note 27-28.

38. Plin., N.H. 3.23.129. 
La più antica è, probabilmente, quella relativa a Lucio Manlio Acidino, membro del collegio triumvirale che fondò la città nel 181 a. C. ${ }^{39}$ :

L(ucius) Manlius L(uci) f(ilius)

Acidinus triu $(m)$ vir

Aquileiae coloniae

deducundae $e^{40}$.

Al riguardo, confermerei quanto già proposto altrove: cioè che l'epigrafe non sia pertinente alla fase iniziale della colonia, ma debba inquadrarsi, per motivi paleografici, nella seconda metà del II secolo a. C. e interpretarsi in chiave commemorativa, cioè quale celebrazione postuma del triumviro ${ }^{41}$ : primo esempio di un' attitudine a coltivare la storia della comunità di Aquileia che proseguì fino al Tardo Impero, come ho sottolineato nel Convegno sui paesaggi epigrafici tenutosi recentemente a Santander ${ }^{42}$.

Del tutto diversa è la situazione della base di Tito Annio (Losco), membro della collegio triumvirale che guidò un supplementum coloniario nel 169 a. C. ${ }^{43}$ (Fig. 6):

T(itus) Annius T(iti) f(ilius) tri(um)vir.

Is hance aedem

faciundam dedit

dedicavitque legesq(ue)

39. Liv., 39.55.5-6 (183 a. C.); 40.34.2-3 (181 a. C.). Un esame prosopografico di questo triumvirato in G. Bandelli, "Aquileia colonia Latina dal senatus consultum del 183 a. C. al supplementum del 169 a. C.", in G. Cuscito (Ed.), Aquileia dalle origini alla costituzione del Ducato longobardo. Storia - Amministrazione Società, Atti della XXXIII Settimana di Studi Aquileiesi (2002), Trieste 2003, 49-78, in part. 60-61.

40. CIL I'2 621 (e p. 919); ILLRP 324; Imagines 143; InAq 27; Lettich, op. cit., 2003, 9, nr. 1; cfr. Bandelli, op. cit., 1988, p. 97, nr. 1 (dove altre indicazioni). Riproduzioni del monumento anche in G. Bandelli, "Aquileia colonia Latina", in J. L. Jiménez Salvador e A. Ribera i Lacomba (Edd.), Valencia y las primeras ciudades romanas de Hispania, Valencia 2002, 57-69, in part. 58, in M. Chiabà, "Dalla fondazione all' età tetrarchica", in F. Ghedini, M. Bueno, M. Novello (Edd.), Moenibus et portu celeberrima. Aquileia: storia di una città, Roma 2009, 7-22, in part. 9, in C. Zaccaria, "Aquileia e l' Impero Romano. Da emporio della X Regio a capitale della Venetia et Histria", in L. Fozzati (Ed.), Aquileia, patrimonio dell' umanità, Udine 2010, 65-87, in part. 72, e in G. Bandelli, "Ancora sul paesaggio epigrafico del Foro di Aquileia repubblicana", in J. M. Iglesias Gil e A. Ruiz Gutiérrez (Edd.), Paisajes epigráficos de la Hispania romana. Monumentos, contextos, topografías, [Atti del Convegno internazionale (Santander 2013)], Roma 2013, 187, fig. 3.

41. Bandelli, op. cit., 1988, 74-75 e G. Bandelli, "Roma e la Venetia orientale dalla guerra gallica (225-222 a. C.) alla guerra sociale (91-87 a. C.)", in G. Cresci Marrone e M. Tirelli (Edd.), Vigilia di romanizzazione. Altino e il Veneto orientale tra II e I sec. a. C., Atti del Convegno (Venezia 1997), Roma 1999, 290, nota 49 (dove altre indicazioni).

42. Bandelli, loc. cit., 2013, 183-203, in part. 188 , note 19-20 e 198, note 87-88.

43. Liv. 43.17.1. Un esame prosopografico di questo triumvirato in Bandelli, loc. cit., 2003, 64-67. 
composivit deditque,

senatum ter coptavit ${ }^{44}$.

Datandosi, ancora sul piano paleografico (per le stringenti analogie con il titulus metrico di Gaio Sempronio Tuditano, che abbiamo esaminato in precedenza), fra gli Anni 130 e 120 a. C. ${ }^{45}$, pure l' iscrizione di Tito Annio (Losco) è da interpretare in senso non autocelebrativo od onorario ma commemorativo, presentandosi come un elogium di carattere non complessivo ma focalizzato su Aquileia, per la cui esegesi rimando nuovamente alla mia relazione di Santander ${ }^{46}$.

Con il documento successivo, pure di Aquileia, entriamo invece nel I secolo a. C. Si tratta dell'epigrafe, da tempo scomparsa, di un Aurelio Cotta (Fig. 7), cui Carlo Rodari, auctor della fine del XVIII secolo, attribuisce il prenome Marcus, ripreso nel CIL:

$M(\operatorname{arco})$ Aurelio $M(\operatorname{arci}) f($ ilio $)$

$\left[\right.$ C]ottae pro co $(n) s(\text { ule })^{47}$;

mentre il suo più giovane contemporaneo Girolamo Asquini e una scheda confluita successivamente nei materiali epigrafici di un altro studioso friulano, Jacopo Pirona, riproducono un testo mancante a sinistra quanto meno del prenome:

[- (- - )] Aurelio M(arci) f(ilio)

$[\text { C]ottae (?) pro co(n)s(ule })^{48}$.

44. C. Zaccaria, "La base di T. Annius Luscus", in Aquileia Nostra, 67, 1996, cc. 179-184; AÉ 1996, 685; F. Maselli Scotti e C. Zaccaria, "Novità epigrafiche dal Foro di Aquileia. A proposito della base di T. Annius T. f. tri. vir", in G. Paci (Ed.), Epigrafia romana in area adriatica, Actes de la IX Rencontre franco-italienne sur l'épigraphie du monde romain (Macerata 1995), Pisa - Roma 1998, 113-159, in part. 117, fig. 1, 4 (F. Maselli Scotti e C. Zaccaria) e 130-143, fig. 5 (C. Zaccaria); AÉ 1998, 17; Lettich, op. cit., 2003, 34, nr. 31. Riproduzioni del monumento anche in Bandelli, loc. cit., 2003, 59, in Chiabà, loc. cit., 2009, 11, in Zaccaria, loc. cit., 2010, 73, fig. [1], in Bandelli, loc. cit., 2013, 188, fig. 4. Per un riesame sistematico del documento cfr., da ultimo, C. Zaccaria, "T. Annius T. f. tri( um) vir e le prime fasi della colonia latina di Aquileia. Bilancio storiografico e problemi aperti", in Hoc quoque laboris praemium. Scritti in onore di Gino Bandelli, Polymnia. Scritti di Storia romana 3, Trieste 2014, 519-552.

45. Bandelli, loc. cit., 1999, 290.

46. Bandelli, loc. cit., 2013, 188-189.

47. CIL V, 863 (e p. 943); CIL I², 739 (e p. 943); InAq 26.

48. Cfr. i commenti del CIL e delle InAq, con discordanze di lettura delle versioni registrate dai vari auctores [a differenza del Mommsen, CIL, il Brusin, InAq, propone comunque un testo integro, con scioglimento diverso del titolo magistratuale: M. Aurelio M. f. / Cottae proco(n)suli]. Sul Rodari (? - ?): CIL V, p. 81. Sull'Asquini (1762-1837), da ultimo: C. Scalon, C. Griggio, U. Rozzo (Edd.), Nuovo Liruti. Dizionario Biografico dei Friulani, 2. L'età veneta, Udine 2009, [I], 320-322 (P. Pastres). Sul Pirona (1789-1870), da ultimo: C. Scalon, C. Griggio, G. Bergamini (Edd.), Nuovo Liruti. Dizionario Biografico dei Friulani, 3. L'età contemporanea, Udine 2011, [III], 2775-2780 (G. Frau). 
863 Aquileiae rep. in effossionibus a. 1788.
$M \cdot A V R E L I O \cdot M \cdot F$ Rodari n. 7; Pironae sche- COTTAE $\cdot \mathrm{PRO} \cdot \mathrm{COS}$ cos. u. c. 690 dium a.1788 n.18; Asquini ms. apud Pironam. 1 ... Vrelio-M.F Pir. sched. et Asq. - 2 cottae Asq. Non caret suspicione utpote coniuncta aliquatenus cum fraudibus Asquinianis. Verum tamen et nomina recte se habent et in titulo dedicato Cottae post annum consulatus antequam fasces deponeret ferri potest, quod solus pro- consulis honor enuntiatur.

Fig. 7. $D a C I L V$

Vanna Vedaldi Iasbez ha dimostrato in modo convincente che il personaggio non può essere Marco Aurelio Cotta, console nel 74 a. C., che dal 73 al 70 esercitò il proconsolato nella Bitinia e nel Ponto, ma dev' essere Gaio Aurelio Cotta, console nel 75 a. C., che nel 74 fu proconsole appunto nella Gallia Citerior, dove condusse alcune operazioni militari che Cicerone giudicava insignificanti ma che gli valsero un trionfo, peraltro non celebrato a causa della sua morte prematura. Che la base, o una delle basi, di tali attività sia stata in Aquileia è dunque altamente probabile e spiegherebbe la collocazione di una statua in onore del magistrato nella colonia Latina divenuta nel 90 a. C. municipium civium Romanorum ${ }^{49}$. Per ulteriori considerazioni rinvio, una volta di più, al mio intervento di Santander ${ }^{50}$.

Tutti i documenti esaminati fino a questo punto si riferiscono a magistrati la cui presenza e le cui iniziative nell' àmbito della provincia furono limitate nel tempo. Del tutto differente risulta il rapporto con la Gallia Cisalpina di Cesare. Anche a voler prescindere dal ruolo avuto da lui nelle prime fasi della causa Transpadanorum, cioè della pressione esercitata dalle comunità locali (almeno dagli Anni Sessanta) per acquistare la cittadinanza romana ${ }^{51}$, resta

49. V. Vedaldi Iasbez, "Magistrati romani ad Aquileia in età repubblicana”, in Mirabella Roberti [(Ed.)], op. cit., 1989, 103-110, seguita da Càssola, loc. cit., 1991, 44; V. Vedaldi Iasbez, "Aquileia: dalla seconda guerra istrica all' età postsillana”, in Cuscito (Ed.), op. cit., 2004, 139-141.

50. Bandelli, loc. cit., 2013, 191, note 45-46.

51. Chevallier, op. cit., 1979, 99-100; R. F. Rossi, “Aquileia in età cesariana ed augustea”, in Cuscito (Ed.), op. cit., 2004, 155-176, in part. 158-163 = R. F. Rossi, Scritti vari sulla Decima Regio con altri saggi di argomento giuliano, Trieste 2008, 351-369, in part. 354-359. 
da considerare il suo proconsolato, esteso fin dall' inizio all' Illirico e successivamente anche alla Gallia Narbonense, ch'ebbe la durata senza precedenti di due quinquenni (58-49 a. C.) ${ }^{52}$.

Da tale punto di vista può sorprendere che le notizie certe e localizzate di soggiorni del governatore nella Gallia Cisalpina - dove pure dedusse la colonia (Latina?) di Novum Comum $^{53}$ e costituì, forse, dei municipia civium Romanorum (come Verona) e, sicuramente, dei centri di statuto diverso (come Forum Iulii) o discusso (come Iulium Carnicum) ${ }^{54}$ - siano alquanto rare; ma la cosa trova spiegazione, almeno in parte, nel fatto che tanto nel De bello Gallico quanto nelle altre fonti sul periodo prevale, con poche eccezioni, l' interesse per le vicende militari svoltesi nella provincia transalpina. Sappiamo, ad ogni modo, che a Mediolanum fu ospite, in data imprecisabile, di un Valerius Leon ${ }^{55}$ e che a Verona lo era, abitualmente, del padre di Catullo ${ }^{56}$. Ad Aquileia, luogo degli hiberna delle sue legioni ${ }^{57}$ e sede certa di uno dei conventus iuridici ${ }^{58}$, risiedette, senza dubbio, più volte e a lungo. I dati concreti al riguardo si riducono però a due: un passo dell'orazione In Vatinium di Cicerone, che documenta la sua presenza nella città durante l' inverno del 57-56 a. C. ${ }^{59}$; e l'epigrafe di Salona relativa alla composizione, avvenuta nel medesimo periodo, cioè il 3 marzo del 56 a. C., della vertenza pertinente a varie comunità dell' Illirico, alla quale già si è accennato ${ }^{60}$. Nel $54 \mathrm{a}$. C. fu dap-

52. Chevallier, op. cit., 1979, 103-114.

53. Novum Comum (59 o 58 a. C.): fonti e bibliografia in Luraschi, op. cit., 1979, 401-506. L' ipotesi che la colonia di Iulia Concordia sia di fondazione cesariana, formulata in V. Vedaldi Iasbez, La Venetia orientale e l'Histria. Le fonti letterarie greche e latine fino alla caduta dell' Impero romano d' Occidente, Roma 1994, 303-306, sembra riferirsi agli Anni Quaranta a. C.

54. Verona (fine degli Anni Cinquanta a. C. ?): E. Buchi, "Porta Leoni e la fondazione di Verona romana", in Museum Patavinum, 5, 1987, 13-45; E. Buchi, Venetorum angulus. Este da comunità paleoveneta a colonia romana, Verona 1993, 41. Forum Iulii (56 a. C.?): R. F. Rossi, "Epigrafia romana di Cividale”, in Mirabella Roberti (Ed.), Studi cividalesi, Atti della giornata di studio (1974), Udine 1975, 23-40, in part. 31-37 = R. F. Rossi, Scritti di storia romana, Trieste 1996, 203-206 = Rossi, op. cit., 2008, 56-61. Iulium Carnicum: G. L. Gregori, "Vecchie e nuove ipotesi sulla storia amministrativa di Iulium Carnicum e di altri centri alpini", in G. Bandelli e F. Fontana (Edd.), Iulium Carnicum, centro alpino tra Italia e Norico dalla protostoria all'età imperiale, Atti del Convegno (Arta Terme - Cividale 1995), Roma 2001, 159-188, in part. 165, nota 36 (municipium); F. Mainardis, Iulium Carnicum. Storia ed epigrafia, Trieste 2008, 36-37 (dallo status quaestionis della studiosa risulta che l' ipotesi prevalente è che si trattasse di un castellum).

55. Plut., Caes. 17.9.

56. Suet., Div. Iul. 73.4.

57. Il primo dato al riguardo è offerto da Caes., $B$. G. 1.10.3: ... ipse in Italiam magnis itineribus contendit duasque ibi legiones conscribit et tres, quae circum Aquileiam hiemabant, ex hibernis educit....

58. La maggior parte delle notizie al riguardo (cfr. supra, nota 13) non sono però localizzate con precisione.

59. Cic., In Vat., 16.38.

60. Cfr. supra, nota 14. 
prima a Ravenna, dove incontrò Marco Licinio Crasso ${ }^{61}$, poi a Luca, dove rinnovò gli accordi con quest' ultimo e Gneo Pompeo ${ }^{62}$.

Di tutti i soggiorni cesariani elencati non resta traccia a livello epigrafico cisalpino. L' unica iscrizione che potrebbe interessare il nostro discorso, per la contiguità con Ravenna, centro non lontano dal confine provinciale, dove il proconsole risiedette alla vigilia della marcia su Roma, è quella di una base mutila di Ariminum, la prima città dell' Italia dopo il Rubicone ${ }^{63}$. Giancarlo Susini la restituisce così:

$$
\begin{aligned}
& {[C(\text { aio }) \text { Mar }] \text { io } C(\text { ai }) f(\text { ilio })} \\
& [\text { tr(ibuno }) \text { pl(ebis }), c o(n) s(u l i)], \text { pro co }(n) s(u l i) \\
& {[\text { Arpin }] \text { ati }^{64} \text {. }}
\end{aligned}
$$

Secondo lo studioso dovrebbe trattarsi, poiché dal punto di vista paleografico il titulus gli appare databile «attorno alla metà del I secolo a. C.»" ${ }^{65}$, dell innalzamento o, eventualmente, del ripristino di una statua di Gaio Mario, promosso dalle autorità locali in onore di Cesare $^{66}$. Che Ariminum sia stato uno degli epicentri delle lotte fra mariani e sillani è noto; e l'esistenza di una $\lambda_{\iota} \theta i v \eta$ cik $\omega \dot{v} v$ del capo dei populares nella vicina Ravenna è ricordata da Plutarco ${ }^{67}$. Ma l' identificazione del titolare del monumento riminese con Gaio Mario è tuttora sub iudice ${ }^{68}$; tanto più, aggiungo, che la facies paleografica sembra ormai al di là della tradizione repubblicana.

La presenza crescente di comunità o di singoli individui dotati della piena cittadinanza - dalle originarie coloniae civium Romanorum alle coloniae Latinae divenute municipia nel 90 a. C. (Lex Iulia) ${ }^{69}$ e ai notabili dei centri indigeni promossi grazie al ius adipiscendae civitatis

61. Cic., Ad fam., 1.9.9 = Cicero: Epistulae ad familiares, Edited by D. R. Shackleton Bailey, vol. I, Cambridge 1977, nr. 20, 63-64 e 310 (dicembre del 54 a. C.).

62. Oltre alla testimonianza ciceroniana cfr. Plut., Caes. 21.5 e Suet., Div. Iul. 24.1.

63. Ravenna: Caes., B. C. 1.5.5. Rubicone: cfr. supra, nota 2.

64. G. Susini, "La dedica a Caio Mario nel foro di Rimini”, in Studi Romagnoli, 13, 1962, 175-190; AÉ 1965, 284; CIL I², 2984, Tabulae, 30, 4.

65. Susini, loc. cit., 1962, 178.

66. Susini, loc. cit., 1962, 190.

67. Ariminum: App., B. C., 1.67 .308 (87 a. C.); 87.396 (82 a. C.); 90.415; 91.422 (82 a. C.); Cic., Verr., 2.1.14.36 (81 a. C.); Chevallier, op. cit., 1979, 94-95. Ravenna: Plut., Mar., 2.1; F. Rebecchi, "Una testa in calcare del Museo Nazionale di Ravenna e il problema cronologico del cd. Bruto Capitolino", in Römisches Porträt. Wege zur Erforschung eines gesellschaftlichen Phänomens, Wissenschaftliche Konferenz (Berlin 1981), Wissenschaftliche Zeitschrift der Humboldt-Universität zu Berlin. Gesellschafts- und Sprachwissenschaftliche Reihe, 31, nr. 2-3, 1982, 261-264, 371-374, figg. 139-146.

68. A. Donati, "Ascesa al senato e rapporti con i territori di origine. Italia. Regio VIII (Aemilia)", in Epigrafia e ordine senatorio, Atti del Colloquio Internazionale AIEGL (Roma 1981), Roma 1982, vol. 2, 302-308, in part. 305: ?? [- - - ]ius C. f:; CIL I², 2984: commento.

69. Cic., Pro Balbo 8.21: ... ipsa denique Iulia, qua lege civitas est sociis et Latinis data; Gell., 4.4.3: ... civitas universo Latio lege Iulia data est. 
per magistratum acquisito nell' 89 a. C. (Lex Pompeia) ${ }^{70}$ - determinò l' ultimo aspetto del fenomeno che stiamo indagando: quello dell' ingresso nel Senato della Repubblica di esponenti delle regioni del Nord.

Per la Cispadana occidentale non abbiamo attestazioni così precoci ${ }^{71}$; per quella orientale i dati sono molto sporadici ${ }^{72}$.

Quanto invece alla Transpadana, dalle ricerche prosopografiche di Géza Alföldy ricaviamo l'esistenza possibile, probabile o certa di senatori eporediesi ${ }^{73}$, bresciani ${ }^{74}$, veronesi ${ }^{75}$, patavini $^{76}$, concordiesi ${ }^{77}$ e aquileiesi ${ }^{78}$ del periodo tardo-repubblicano o primo-augusteo.

Tra quelli probabili o certi ricordati da epigrafi locali sono di particolare interesse, oltre al veronese (?) $Q$ (uintus) Poblicius $Q$ (uinti) f(ilius) ${ }^{79}$, tre aquileiesi: $C$ (aius) Appulleius $M($ arci) f(ilius) Tappo, $M$ (arcus) Fruticius $M$ (arci) f(ilius) e C(aius) Lucilius $C($ ai $) f(\text { ilius })^{80}$.

I monumenti relativi al primo e al terzo di questi ultimi, diversamente da quanto ritiene il Maestro ungherese, che propende, con qualche dubbio, per una loro dimensione funera-

70. Ascon., In Pisonianam, p. 3 Clark $1907=$ p. 12 Stangl 1912 = p. 5 Giarratano 1920: Pompeius enim non novis colonis eas [le comunità indigene] constituit sed veteribus incolis manentibus ius dedit Latii, ut possent habere ius quod ceterae Latinae coloniae, id est ut petendo magistratus civitatem Romanam adipiscerentur.

71. G. Alföldy, "Senatoren aus Norditalien. Regiones IX, X und XI", in Epigrafia e ordine senatorio, op. cit., 1982, vol. 2, 309-368, in part. 323-329 = riedizione con aggiunte, G. Alföldy, "Die Eliten im römischen Norditalien. Versuch einer Synthese", in G. Alföldy, Städte, Eliten und Gesellschaft in der Gallia Cisalpina. Epigraphisch-historische Untersuchungen, Stuttgart 1999, 259-341, in part. 274-283.

72. Donati, loc. cit., 1982, 305: ?? [- - - lius C. f.; 306: ?? T. Fadius (Gallus); ?? (Ti.) Tisienus Gallus; ? C. Cassius Parmensis; 307: ?? C. Antistius Reginus; ?? L. Caninius Gallus, tr. pl. 56 a. C.; ?? L. Caninius Gallus, cos. ord. 37 a. C.

73. Alföldy, loc. cit., 1982, 359, nr. ? 1 = Alföldy, loc. cit., 1999, 329, nr. ? 1.

74. Alföldy, loc. cit., 1982, 346, nrr. 1, ?? 2 = Alföldy, loc. cit., 1999, 308, nrr. 1, ?? 2.

75. Alföldy, loc. cit., 1982, 340-341, nrr. ?? 1, ?? 2, ? 3, ?? 4, ?? 5, ?? 6, ? 7 = Alföldy, loc. cit., 1999, 300-301, nrr. ?? 1, ?? 2, ? 3 , ?? 4 , ?? 5 , ?? 6, ? 7 .

76. Alföldy, loc. cit., 1982, 336, nr. ? 1 = Alföldy, loc. cit., 1999, 294, nr. ? 1.

77. Alföldy, loc. cit., 1982, 333, nr. ? 1 = Alföldy, loc. cit., 1999, 289-290, nr. ? 1.

78. Alföldy, loc. cit., 1982, 331-332, nrr. ?? 1, ? 2, 3, ? 4, ? 5 = Alföldy, loc. cit., 1999, 285-287, nrr. ?? 1, ? 2, 3, ? 4, ? 5 .

79. $A E ́$ 1965, 148; Alföldy, op. cit., 1984, 132, nr. 212; inoltre: Alföldy, loc. cit., 1982, 341, nr. ? $7=$ Alföldy, loc. cit., 1999, 301, nr. ? 7.

80. C. Appulleius M.f. Tappo: CIL I², 814 (e p. 954); ILLRP 436; inoltre: Alföldy, loc. cit., 1982, 331332, nr. 3 = Alföldy, loc. cit., 1999, 286, nr. 3; Bandelli, op. cit., 1988, 163, nr. 38. M. Fruticius M. f. pr(aetor), aed(ilis), $\operatorname{tr}$ (ibunus) pl(ebis) e i suoi familiari (iscrizione funeraria): CIL I², 826 (e p. 956); ILLRP 440; inoltre: Alföldy, loc. cit., 1982, 332, nr. ? 4 = Alföldy, loc. cit., 1999, 286, nr. ? 4; Bandelli, op. cit., 1988, 162, nr. 36. C. Lucilius C. f:: CIL I², 2204 (e p. 956, Tabulae 31, 1); inoltre: Alföldy, loc. cit., 1982, 332, nr. ? 5 = Alföldy, loc. cit., 1999, 286-287, nr. ? 5; Bandelli, op. cit., 1988, 161-162, nr. 35. 
814 basis magna quadrata. Rep. Aquileiae a. 1726 ad pontem Monasterii.

$$
\begin{array}{cc}
\text { C.APPVLLEIVS } \\
\text { M.F.TAPPO } \\
\text { PR.AED.TR.PL.O } \\
\text { IVDEX } \\
5 \text { OVAESITIONIS } \\
\text { RERVM.CAPITAL }
\end{array}
$$

CIL. V 862. - Exhibent Beretta ms. duobus exemplis; Bertoli p. 300 n. 419 ; inde posteriores. - Orelli 3827; Dessau 906. 1 AppVlleivs Bert., appyleivs Ber. 1. 2. - 5 QVAestionis Ber. 6 RER - Ber. 2.

Apparet honores legitimos ordine recenseri, subici his iudicis quaestionis officium tamquam extraordinarium; quamquam secundum temporis ordinem sine dubio interponendum fuit inter praeturam et aedilitatem (Mommsen), cf. Staatsrecht II ${ }^{3}$ p. 555. 586 sqq.

Fig. 8. Da CIL I $\mathrm{I}^{2}$

ria $^{81}$, potrebbero essere invece delle basi di statue equestri, provenienti dal foro di Aquileia ${ }^{82}$. Nel caso della prima, viste le sue misure, che sono conosciute ( $\mathrm{cm} 119 \times 68 \times 238)$ anche se il blocco è perduto, la conclusione pare certa ${ }^{83}$. Eccone il testo (Fig. 8):

C(aius) Appulleius

M(arci) f(ilius) Tappo

pr(aetor), aed(ilis), $\operatorname{tr}($ ibunus) pl(ebis), q(uaestor),

iudex

quaesitionis

rerum capital(ium $)^{84}$.

81. Appulleius: Alföldy, loc. cit., 1982, 331-332, nr. 3 («... vermutlich... Grabinschrift») = Alföldy, loc. cit., 1999, 286, nr. 3 («... Grabinschrift, obwohl man auch an die Inschrift auf einem Statuenpostament denken kann»). Lucilius: Alföldy, loc. cit., 1982, 332, nr. ? 5 («... vermutlich... Grabinschrift...») = G. Alföldy, loc. cit., 1999, 286-287, nr. ? 5 («... vermutlich... Grabinschrift...»).

82. Per una rassegna completa degli esemplari aquileiesi di analoga struttura (da quattro a otto) cfr. Bandelli, loc. cit., 2013, 191-195.

83. Bandelli, loc. cit., 2013, 191-192. Meno sicura, invece, quella relativa al monumento di C. Lucilius C. f: Bandelli, loc. cit., 2013, 193-194.

84. Cfr. supra, note 78 e 80. 
Dal complesso delle fonti, letterarie ed epigrafiche, disponibili sembrano quindi emergere le seguenti conclusioni generali: che nella Gallia Cisalpina le «ciudades del poder» furono per tutto il II secolo a. C. dei centri coloniari (come Luna, Parma, Aquileia), sedi anche di manifestazioni (come l'evergetismo «trionfale» e le iscrizioni «trionfali») riservate in precedenza alla capitale della Repubblica; mentre nel I secolo a. C. e soprattutto nell' età cesariana, ferma restando la posizione di privilegio di Aquileia, emersero come luoghi di residenza di esponenti del governo centrale anche delle comunità indigene (da Mediolanum a Verona e Ravenna). 